\title{
Effects of surface roughness and film thickness on the adhesion of a bioinspired nanofilm
}

\author{
Z. L. Peng and S. H. Chen* \\ LNM, Institute of Mechanics, Chinese Academy of Sciences, Beijing 100190, China \\ (Received 13 January 2011; revised manuscript received 4 March 2011; published 16 May 2011)
}

\begin{abstract}
Inspired by the gecko's climbing ability, adhesion between an elastic nanofilm with finite length and a rough substrate with sinusoidal roughness is studied in the present paper, considering the effects of substrate roughness and film thickness. It demonstrates that the normal adhesion force of the nanofilm on a rough substrate depends significantly on the geometrical parameters of the substrate. When the film length is larger than the wavelength of the sinusoidal roughness of the substrate, the normal adhesion force decreases with increasing surface roughness, while the normal adhesion force initially decreases then increases if the wavelength of roughness is larger than the film length. This finding is qualitatively consistent with a previously interesting experimental observation in which the adhesion force of the gecko spatula is found to reduce significantly at an intermediate roughness. Furthermore, it is inferred that the gecko may achieve an optimal spatula thickness not only to follow rough surfaces, but also to saturate the adhesion force. The results in this paper may be helpful for understanding how geckos overcome the influence of natural surface roughness and possess such adhesion to support their weights.
\end{abstract}

DOI: 10.1103/PhysRevE.83.051915

PACS number(s): 87.85.Qr, 68.37.-d, 87.80.-y

\section{INTRODUCTION}

Few surfaces are smooth at the atomic level, and even highly polished surfaces possess surface roughness on many different length scales. Surface roughness may have a significant influence on the adhesion between an elastic solid and a hard substrate, e.g., polymer/metal junction and biological adhesion. Initially, this topic was studied by Fuller and Tabor [1] in which they developed a simple model based on the assumption of surface asperity following a Gaussian distribution, and the overall contact force was obtained by applying the Johnson-Kendall-Roberts contact theory [2] to each individual asperity. It was found that relatively small surface roughness could reduce or even remove adhesion. This perspective was accepted for a long time until Briggs and Briscoe [3] reported their experimental results in which rubber could stick to a rigid slightly rough surface much better than to a relatively smooth one. Fuller and Roberts [4] confirmed such an effect using very soft rubber with a rolling resistance experiment. In order to find the mechanisms, many theoretical and numerical models have been developed in which the effect of surface roughness on adhesion is considered [5-13]. For example, Persson and Tosatti [8], Persson [9], Persson and Gorb [14], and Palasantzas and De Hosson [10,11] analyzed adhesion of randomly rough surfaces on many different length scales, which was described as a self-affine fractal. It was shown that adhesion of an elastic solid to a rough substrate involves competition between the attractive adhesion energy $\Delta \gamma A$, which mainly results from the regions where the two solids are in atomic contact at the interface, and the repulsive elastic energy $U_{\mathrm{el}}$, associated with the bending of the surface of the elastic solid. The effective interfacial energy can be obtained as $\Delta \gamma_{\text {eff }} A_{0}=\Delta \gamma A-U_{\mathrm{el}}$, where $A_{0}$ is defined as a nominal contact area and $A$ is the true atomic contact area. When the attractive adhesion energy $\Delta \gamma A$ is larger than the elastic energy $U_{\mathrm{el}}$, the elastic solid/film will deform spontaneously to fill out the substrate cavities, and complete

*chenshaohua72@ @otmail.com contact will occur [15]. A similar complete contact condition had also been derived by Majidi and Fearing [16] in which a three-dimensional adhesion of an elastic plate adhering to a sphere is investigated, and stretching of the plate is considered in order to adhere to the rigid sphere. In the case of a plane strain complete contact, if the increased interfacial energy by the difference of the real contact area and the nominal one is much larger than the stored elastic bending energy, adhesion force may be improved by the surface roughness, which may explain the phenomenon found in Ref. [3].

One can see that most of the above studies were focused either on bulk stiff materials in which the real contact area may be a small fraction of the nominal one, thus, leading to a decreasing adhesion or on a complaint film in which surface roughness will increase the real contact area. Simultaneously, part of strain energy should be stored in films due to film bending, thus, yielding a slightly enhanced adhesion or a monotonically decreasing one.

However, Huber et al. [17] recently measured the peeling force of a single gecko's spatula that made contact with different rough surfaces at a peeling angle of $\theta=90^{\circ}$ and found a very interesting experimental observation on the effect of surface roughness on adhesion: The adhesion force of a gecko's spatula does not increase or decrease monotonically with surface roughness but is strongly reduced at an intermediate rms roughness ranging from 100 to $300 \mathrm{~nm}$, above or below which the adhesion forces increase with increasing or decreasing surface roughness. Actually, friction measurements on flies and beetles, walking on surfaces with well-defined surface roughness, showed the same phenomena. A minimum in friction force was identified for a certain range of surface roughness, varying from $0.3 \mu \mathrm{m}$ to $1 \mu \mathrm{m}$ for the beetle Gastrophysa viridula [18] and for the fly Musca domestica [19].

The above phenomena cannot be explained by the existing literature, including the theoretical model considering multiscale surface roughness presented by Persson and Gorb [14]. What is the reason? In fact, all the theoretical adhesion models for thin films considering the effect of surface roughness are focused on films with infinite length, which should predict 
different mechanical phenomena for a thin film with a finite length scale, such as Peng et al. [12]. Naturally, a gecko's spatula has a finite size, and the adhesion of a spatula pad is similar to a nanofilm with a finite length scale adhering to a substrate [20,21]. The peeling force that involves competition between van der Waals forces and elastic deformation of the pad [22] can be derived from the Kendall model [23],

$$
P=\frac{\Delta \gamma}{1-\cos \theta}+\text { elastic energy term, }
$$

where $\Delta \gamma$ is the interfacial energy per unit area of two flat contacting surfaces and $\theta$ is a peeling angle. When a spatula pad adheres to a rough surface, the peeling force of the spatula pad detaching from the rough surface can be derived by replacing the interfacial energy $\Delta \gamma$ with the effective interfacial energy $\Delta \gamma_{\text {eff }}$.

In order to explain the interesting observations reported by Huber et al. [17], a theoretical model of an elastic nanofilm in adhesive contact with a rough surface is established in the present paper. The nanofilm has a finite length scale similar to a gecko's spatula. Compared to the random surface roughness [8], the surface roughness in the present paper is represented by a simple sinusoidal profile, which is similar to a cosine corrugation used by Zilberman and Persson [24] and is often used in Ref. [25] without losing the problem's essence. Effects of the wavelength of the surface roughness, the ratio of the amplitude to the wavelength, as well as the thickness of a nanofilm on adhesion are mainly investigated. The results provide a different understanding of biological adhesion.

\section{THEORETICAL MODEL OF A FINITE NANOFILM IN ADHESIVE CONTACT WITH A ROUGH SURFACE}

\section{A. Effective interfacial energy}

A partial contact model has been investigated by Persson [9] in which it is found that the effective interfacial energy decreases or increases and then decreases with increasing surface roughness. However, this result cannot give a reasonable demonstration on the experimental observation found by Huber et al. [17]. A plane strain complete contact model is established in the present paper as shown in Fig. 1 in which an elastically flexible nanofilm with small bending stiffness is easy to bend and completely follows the substrate roughness. $E$ and $v$ are the Young's modulus and the Poisson's ratio of the nanofilm, respectively, as well as a finite length $b$. As an example, the surface roughness is assumed to be described by a sinusoidal function as

$$
w=y=a-a \cos (k x),
$$

where $a$ is the amplitude of the roughness, $k=2 \pi / \lambda$ is the wave number, and $\lambda$ is the wavelength.

As mentioned in Ref. [14], adhesion of an elastic solid on a rough substrate involves competition between the attractive adhesion energy, which mainly arises from the region where the two solids are in atomic contact at the interface, and the repulsive elastic energy associated with the bending of the elastic solid so that it comes in direct atomic contact with the substrate. If $b$ is the true atomic contact area and $b_{0}$ is

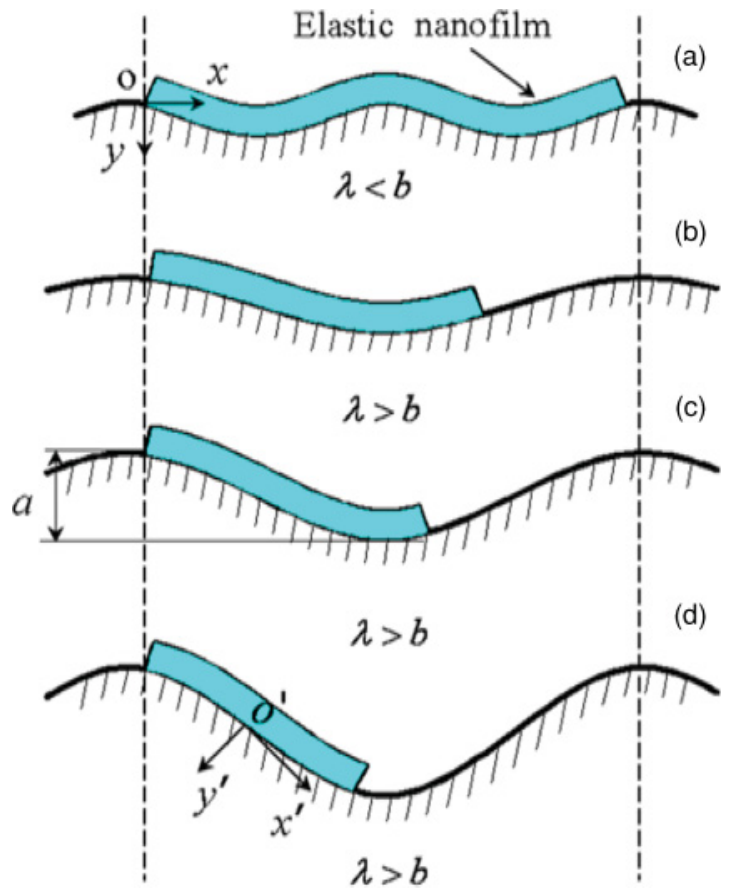

FIG. 1. (Color online) Schematic of a finite-size nanofilm in adhesive contact with a sinusoidal rough substrate. (a) Wavelength $\lambda$ of the surface roughness smaller than the length $b$ of the nanofilm; (b), (c), and (d) $\lambda$ larger than $b$ but with increasing amplitudes.

the nominal contact area between surfaces, then the effective interfacial energy can be written as

$$
\Delta \gamma_{\mathrm{eff}} b_{0}=\Delta \gamma b-U_{\mathrm{el}}
$$

where $\Delta \gamma=\gamma_{1}+\gamma_{2}-\gamma_{12}$ is the change of the interfacial energy (per unit area) when perfect flat surfaces are brought into contact. $U_{\mathrm{el}}$ is only the bending energy necessary to make atomic contact at the interface, which is different from the three-dimensional case of an elastic plate adhering to a sphere [16]. In the three-dimensional case [16], not only the bending energy, but also the stretching energy should be included because a thin elastic plate must bend and must stretch in order to adhere to a rigid sphere. In the present plane strain case, complete contact can be realized by bending without stretching action.

Comparing the wavelength, the amplitude of the surface roughness and the film length leads to different contact cases as shown in Figs. 1(a)-1(d) in which we assume the starting point is located at the peak of the sinusoidal profile. Another case where the peaks of the roughness are making contact with the film is not demonstrated schematically in Fig. 1 since the mechanism is same as the film adhering to the valley of the substrate roughness. Figure 1(a) represents the case with the film length much larger than the wavelength of the surface roughness, and Figs. 1(b)-1(d) describe the cases in which the film length is smaller than the wavelength but with different amplitudes. 
(a) Consider the case with film length $b$ much larger than wavelength $\lambda$ [Fig. 1(a)]. The elastic bending energy can beobtained as

$$
U_{\mathrm{el}}=\frac{E I}{2} \int\left(\frac{d^{2} w}{d x^{2}}\right)^{2} d x,
$$

where $I=h^{3} / 12$ is the inertia moment, $h$ is the thickness, and $w$ is the deflection of the film. Substituting the expression of deflection in Eq. (2) into Eq. (4) leads to

$$
U_{\mathrm{el}}=\frac{E h^{3}}{48} a^{2} k^{4}\left[b_{0}+\frac{\sin \left(2 k b_{0}\right)}{2 k}\right] .
$$

The nominal contact area $b_{0}$ can result from the following formula:

$$
\int_{0}^{b_{0}} \sqrt{1+a^{2} k^{2} \sin ^{2}(k x)} d x=b .
$$

Introducing an adhesion length $\delta=\Delta \gamma_{0} / E$ similar to that in Ref. [14] and dimensionless parameters $\widetilde{a}=a / \lambda, \widetilde{b}=b / \lambda$, $\widetilde{b}_{0}=b_{0} / \lambda, \widetilde{h}=h / \lambda$, and $\widetilde{\delta}=\delta / \lambda$, the normalized effective interfacial energy can be obtained as

$$
\frac{\Delta \gamma_{\mathrm{eff}}}{\Delta \gamma}=\frac{\tilde{b}}{\tilde{b}_{0}}-\frac{(2 \pi)^{4} \tilde{a}^{2} \tilde{h}^{3}}{48 \tilde{b}_{0} \tilde{\delta}}\left[\tilde{b}_{0}+\frac{1}{4 \pi} \sin \left(4 \pi \tilde{b}_{0}\right)\right] .
$$

(b) For the case with the film length smaller than the wavelength of surface roughness, there are three possible complete contact schematics as shown in Figs. 1(b)-1(d). It is convenient for us to calculate the bending energy of the nanofilm under the coordinate system $\left(x^{\prime} o^{\prime} y^{\prime}\right)$ as shown in Fig. 1(d), which can be obtained by rotating the original coordinate system (xoy) with an angle $\theta=\arctan (a k)$. Then, the relationship between the two coordinate systems can be written as

$$
x=x^{\prime} \cos \theta-y^{\prime} \sin \theta, \quad y=x^{\prime} \sin \theta+y^{\prime} \cos \theta .
$$

In the coordinate system $\left(x^{\prime} o^{\prime} y^{\prime}\right)$, one can see that the nominal contact area $b_{0}$ approaches the actual contact area $b$. Thus, we assume $b=b_{0}$ in this case. The deflection of the film in $x^{\prime} o^{\prime} y^{\prime}$ can be calculated from Eq. (2) by the technique of coordinate transformation, which, however, leads to a very complex expression. For simplicity, a quintic polynomial is used to calibrate the deflection of the curved nanofilm in $x^{\prime} o^{\prime} y^{\prime}$. Then, the elastic bending energy $U_{\text {el }}$ can easily be obtained by substituting the quintic polynomial deflection into Eq. (4), which leads to the effective interfacial energy,

$$
\frac{\Delta \gamma_{\mathrm{eff}}}{\Delta \gamma}=1-\frac{U_{\mathrm{el}}}{\Delta \gamma b} \text {. }
$$

The influences of the wavelength and the amplitude of the rough substrate on the effective interfacial energy will be analyzed in Sec. III.

The interfacial energy $\Delta \gamma$ is assumed to be a constant in the above analysis. Actually, the surface energy that is caused by van der Waals force depends on the dimension of a nanofilm when the nanofilm becomes thin enough. Thus, it is reasonable to predict the effect of film thickness on adhesion energy. The size dependence of interfacial energy was first considered thermodynamically by Tolman [26]. Assuming the Tolman length $\alpha$ to be a constant in the nanometer region, Tolman derived the following analytical equation [26]:

$$
\frac{\Delta \gamma(h)}{\Delta \gamma_{0}}=\frac{1}{1+4 \alpha / h}=1-\frac{4 \alpha}{h}+\cdots,
$$

where $\Delta \gamma_{0}$ is an interfacial energy per unit area between two bodies and $h$ is the film thickness. The other form of size-dependent interfacial energy, for example, an exponential mode, can also be found in Ref. [27]. Substituting Eq. (10) into Eqs. (7) and (9) leads to the size-dependent effective interfacial energy when the length of the nanofilm is larger than the wavelength,

$$
\frac{\Delta \gamma_{\text {eff }}}{\Delta \gamma_{0}}=\left(1-\frac{4 \tilde{\alpha}}{\tilde{h}}\right) \frac{\tilde{b}}{\tilde{b}_{0}}-\frac{(2 \pi)^{4} \tilde{a}^{2} \tilde{h}^{3}}{48 \tilde{b}_{0} \tilde{\delta}}\left[\tilde{b}_{0}+\frac{1}{4 \pi} \sin \left(4 \pi \tilde{b}_{0}\right)\right] .
$$

For the case of the nanofilm length smaller than the wavelength, we have

$$
\frac{\Delta \gamma_{\mathrm{eff}}}{\Delta \gamma_{0}}=\left(1-\frac{4 \alpha}{h}\right)-\frac{U_{\mathrm{el}}}{\Delta \gamma_{0} b} .
$$

From above, one can see that only the totally normal adhesion force is considered, which is similar to the theoretical analysis by Persson and Tosatti [8] and Palasantzas and De Hosson [11]. If the peeling case is considered, a modified version of the Kendall peel theory similar to that used by Majidi and Fearing [16] can be utilized to find the relation among the peeling force, peeling angle, and parameters of roughness only by replacing the interfacial energy $\Delta \gamma$ with the effective interfacial energy $\Delta \gamma_{\text {eff }}$ in Eq. (1), under which the work of adhesion for a flat substrate $\Delta \gamma$ may be a function of peeling angle $\theta$ [21] and consists of the contributions of both the normal traction and the tangential one at the interface. However, one of the main motivations of the present paper is to explain the relation of the spatula adhesion force and the surface roughness. The peeling case must be considered because the adhesion of a gecko's spatula is similar to a thin film under peeling, and this will be discussed in Sec. III.

\section{B. Effect of nanofilm thickness on adhesion}

The force arising from molecular interaction between two contact solids determines the adhesion of the contact interface. The effective interaction distance between two molecules is known to be finite, which leads to an asymptotical value of the adhesion force between two contacting solids regardless of the thickness of the solids. It can be inferred that, if the thickness of a film in contact with a substrate is less than the effective interaction distance of molecules, the interfacial adhesion force should increase with an increasing film thickness and asymptotically should tend to be constant until a critical film thickens. Actually, experiments have shown that geckos mainly depend on van der Waals forces to maintain their staying or climbing on a wall [28,29], and the adhesive system is a hierarchical structure with the smallest element called a spatula. Each spatula has a finite size. Especially, the thickness of a spatula is about $5 \mathrm{~nm}$ [20]. Other literature reported that the thickness of a spatula is $5-10 \mathrm{~nm}[14,17,30], 5 \mathrm{~nm}$ may be taken as the lower limit of the thickness of a spatula in the present paper. Questions are still open as to why geckos 
possess such a spatula with such a thickness and whether it is an optimum thickness that nature provides.

Assuming interaction between two adjacent atoms governed by the Lennard-Jones (L-J) 12-6 potentials,

$$
w(r)=4 \varepsilon\left[\left(\frac{\sigma}{r}\right)^{12}-\left(\frac{\sigma}{r}\right)^{6}\right],
$$

where $\varepsilon$ is a parameter determining the depth of the potential well, $\sigma$ is a length scale parameter that determines the position of the minimum potential, and $r$ is atom-to-atom distance [31,32]. We can approximately estimate the value of the parameter $\sigma=r_{0} / \sqrt[6]{2}$ by the balance distance $r_{0}$, which can be obtained from the equilibrium condition $f(r)=\partial w / \partial r=0$. If we take the equilibrium distance between two interacting atoms as $r_{0}=0.3 \mathrm{~nm}$, which yields $\sigma \approx 0.27 \mathrm{~nm}$, with the additional assumption of additivity, the net interaction energy between a molecule and a planar surface of a solid made up of the same molecules will be the sum of its interactions with all the molecules in the body as shown in Fig. 2(a) [31],

$$
\begin{aligned}
w(D) & =2 \pi \rho_{1} \int_{D}^{\infty} d z \int_{0}^{\infty}\left[\frac{4 \varepsilon \sigma^{12} x}{\left(z^{2}+x^{2}\right)^{6}}-\frac{4 \varepsilon \sigma^{6} x}{\left(z^{2}+x^{2}\right)^{3}}\right] d x \\
& =4 \pi \varepsilon \rho_{1}\left(\frac{\sigma^{12}}{45 D^{9}}-\frac{\sigma^{6}}{6 D^{3}}\right),
\end{aligned}
$$

where $\rho_{1}$ is the number of molecules per unit volume in the solid and $D$ is the distance between the single molecule and the planar surface of the semi-infinite body. Therefore, the interaction energy between a nanofilm with thickness $h$ and the planar surface of a semi-infinite body with a separation $D$ can be obtained by the integration of Eq. (14),

$$
\begin{aligned}
W(h)= & 4 \pi \varepsilon \rho_{1} \rho_{2} \iint d x d y \int_{D}^{D+h}\left(\frac{\sigma^{12}}{45 z^{9}}-\frac{\sigma^{6}}{6 z^{3}}\right) d z \\
= & 4 \pi \varepsilon \rho_{1} \rho_{2} A\left[-\frac{\sigma^{12}}{360(D+h)^{8}}+\frac{\sigma^{6}}{12(D+h)^{2}}\right. \\
& \left.+\frac{\sigma^{12}}{360 D^{8}}-\frac{\sigma^{6}}{12 D^{2}}\right],
\end{aligned}
$$

where $\rho_{2}$ is the number of molecules per unit volume in the nanofilm. $A$ is the cross-sectional area of the nanofilm in the
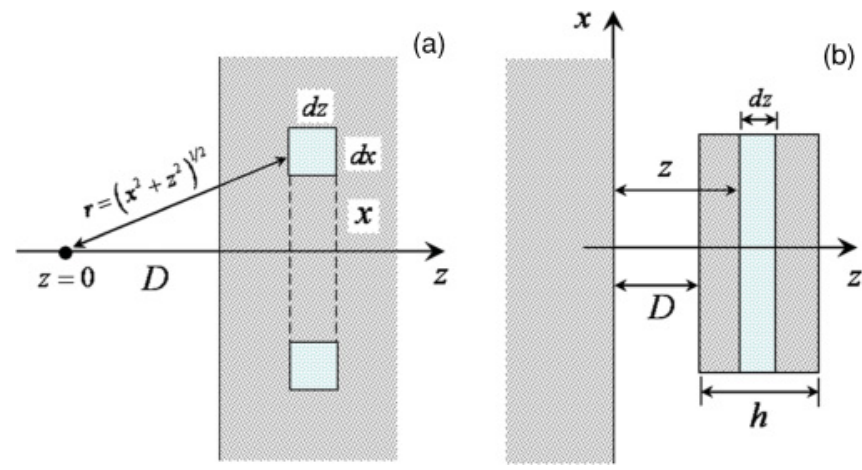

FIG. 2. (Color online) Schematic of obtaining the interaction energy between a finite-size nanofilm and a semi-infinite space. (a) A molecule near a semi-infinite body with distance $D$; (b) a finite-size nanofilm with thickness $h$ near a semi-infinite body with distance $D$. Adopted from Ref. [31]. $x-y$ plane [Fig. 2(b)]. When the film thickness tends to be infinite $(h \rightarrow \infty)$, then the adhesive model becomes the one between two infinite bodies. The adhesion energy $W(h)$ will reduce to a constant $-C / 12 D^{2}$, which is often adopted in literature $[20,33]$. The constant $C$ is a combination of the other parameters, such as $\rho_{1}, \rho_{2}, A, \varepsilon$, and $\sigma$. The corresponding interaction force can be derived as

$$
\begin{aligned}
F(h)= & \frac{\partial W}{\partial D}=4 \pi \varepsilon \rho_{1} \rho_{2} A \\
& \times\left[\frac{\sigma^{12}}{45(D+h)^{9}}-\frac{\sigma^{6}}{6(D+h)^{3}}-\frac{\sigma^{12}}{45 D^{9}}+\frac{\sigma^{6}}{6 D^{3}}\right] .
\end{aligned}
$$

The normalized interaction force between the nanofilm and the infinite body can be rewritten as

$$
\begin{aligned}
\frac{F(h)}{4 \pi A \varepsilon \rho_{1} \rho_{2} \sigma^{3}}= & \frac{1}{45(D / \sigma+h / \sigma)^{9}}-\frac{1}{6(D / \sigma+h / \sigma)^{3}} \\
& -\frac{1}{45(D / \sigma)^{9}}+\frac{1}{6(D / \sigma)^{3}}
\end{aligned}
$$

which gives the relation between the interaction force of a nanofilm in contact with an infinite substrate and the thickness of a nanofilm. The effect of a nanofilm's thickness on the interaction force will be analyzed numerically in Sec. III.

One should note that the L-J potential is a two-body summation method that assumes the force between any two molecules is not affected by the presence of the other nearby molecules so that one can add all the pair potentials of a molecule to obtain its net interaction energy with all the other molecules. However, van der Waals forces are not generally pairwise additive: The force between any two molecules is affected by other molecules [31]. If the pair potential is used to calculate the macroscopic interaction between two bodies, there must be some prerequisite assumptions (a) additivity: The total force can be obtained by the pairwise summation of individual contributions; (b) continuous medium: The summation is replaced by an integration over the volume; (c) constant material properties: The densities $\rho$ and the interaction constants do not vary over the volume of the body [34]. All these assumptions are adopted in the present simple analysis. A more accurate method calculating the interaction between multiatom systems is to use the many-body potential [35-38], which considers the effect of other atoms. However, the problem will become very complicated, although the effect to calculate the interaction with a pair potential instead of a many-body potential is small [39].

\section{RESULTS AND DISCUSSION}

In the present paper, we will use the ratio of amplitude $a$ to wavelength $\lambda$ to define the surface roughness. However, since we have adopted the assumption that the finite nanofilm adhesively contacts the rough substrate completely, this assumption requires that the wavelength of the surface roughness should not be very small. If the surface roughness possesses a small wavelength with small amplitude, the surface will tend to be a flat one. If the surface roughness possesses a small wavelength with large amplitude, complete contact cannot occur. The wavelength $\lambda$ must have a reasonable region in our present 


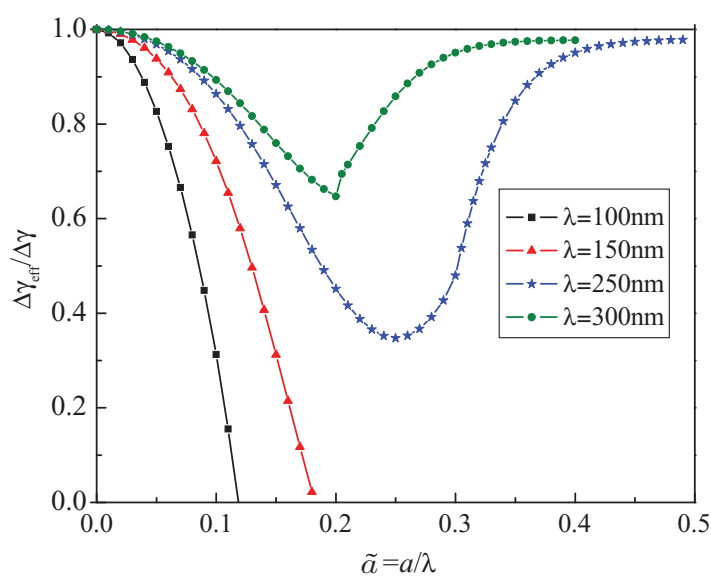

FIG. 3. (Color online) The relation between the normalized effective interfacial energy $\Delta \gamma_{\text {eff }} / \Delta \gamma$ and the surface roughness $a / \lambda$ for cases with different wavelengths $\lambda$, where $\Delta \gamma$ is the interfacial energy for a flat surface case and $a$ is the amplitude of the sinusoidal profile of the surface.

analysis to satisfy the condition of complete contact. The best choice for the magnitude of $\lambda$ is to have the same order as the length of the nanofilm. In the following analysis, the surface roughness is mainly adjusted by the value of amplitude $a$. As for the noncomplete contact case, it will be left to future work.

Figure 3 indicates the effective interfacial energy normalized by the interfacial energy of a flat interface as a function of the substrate roughness, which is expressed by the ratio of amplitude $a$ to wavelength $\lambda$ of the rough surface $a / \lambda$, for a set of values of $b=200, h=5 \mathrm{~nm}, E=2 \mathrm{GPa}, \Delta \gamma=0.01 \mathrm{~J} / \mathrm{m}^{2}$, and different wavelengths. From Fig. 3, one can see that, when the length $b$ of the nanofilm is larger than the wavelength, the effective interfacial energy decreases monotonically with increasing surface roughness, which is consistent with the results of a more physically three-dimensional plausible hemispherical bump model $[1,16]$, while the effective interfacial energy decreases first and then increases if the length of the nanofilm is smaller than the wavelength. The results for a finite-size nanofilm in adhesive contact with a rough surface in this paper is essentially different from that of a bulk material adhering to a rough substrate [1], where the adhesion force decreases monotonically with increasing surface roughness. From above, one can see that all the parameters for the finitesize nanofilm are similar to those for geckos' spatulas [20]. In order to explain the interesting experimental observation by Huber et al. [17], the peeling force of an adhesive pad adhering to a rough surface at a peeling angle, $\theta=90^{\circ}$, must be obtained. Replacing the interfacial energy $\Delta \gamma$ with the effective interfacial energy $\Delta \gamma_{\text {eff }}$ in Eq. (1), the peeling force becomes $P=2 \Delta \gamma_{\mathrm{eff}} /\left(\sqrt{1+2 \Delta \gamma_{\mathrm{eff}} / E h}+1\right)$. Figure 4 demonstrates the peeling force as a function of the effective interfacial energy with $E=2 \mathrm{GPa}$ and $h=5 \mathrm{~nm}$. One can see that the contribution of the term $\Delta \gamma_{\text {eff }} / E h$ is very small so that the peeling force increases almost linearly with an increasing effective interfacial energy, i.e., $P \approx \Delta \gamma_{\text {eff }}$. This result is very consistent with the analysis by Kendall [23] in which it is found that, generally, the contribution of the elastic term may be neglected because the stress in the film is usually

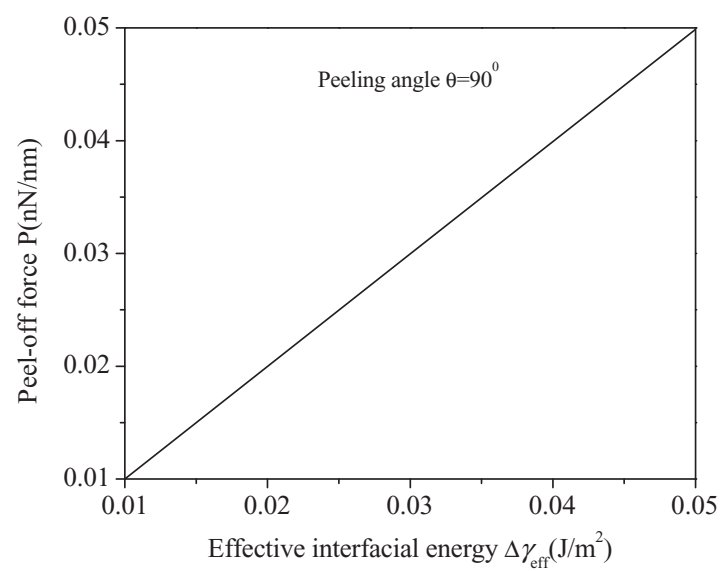

FIG. 4. Peelingforce of a spatula pad adhering to a rough surface as a function of the effective interfacial energy at a peeling angle of $\theta=90^{\circ}$.

much smaller than Young's modulus $E$, and the elastic term becomes significant only when the peeling angle $\theta$ becomes small. At the peeling angle of $\theta=90^{\circ}$, the elastic term is only about $1 \%$ of the other terms and, therefore, is neglected. Thus, it can be inferred that the peeling force at $\theta=90^{\circ}$ should have the same variation tendency as the effective interfacial energy. Therefore, qualitatively, the present results agree well with the experimental observation [17] that the adhesion force of the gecko spatula is strongly reduced at an intermediate roughness. When the surface roughness is small, the nominal contact area is almost equal to the real contact area, which results in less elastic energy stored in the film. A similar explanation should also be true for the case of a surface with substantial roughness (if the wavelength is fixed, the larger the amplitude, the rougher the surface). For the case of a surface with intermediate roughness, more elastic energy stored in the bending film leads to a reduced effective interfacial one and, thus, a reduced adhesion force. The general conclusion can be made that the adhesion behavior of a finite-size nanofilm is different from that of an infinite one due to the size effect.

Although the present results cannot quantitatively compare with the experimental results [17] due to the assumption of an optimal sinusoidal surface profile, the plane strain model, and some undermined parameters $\rho_{1}$ and $\rho_{2}$, the previous works that studied the effect of surface roughness on adhesion with an infinite length nanofilm, including the random roughness model $[8,11,14]$, cannot explain the interesting experimental observation, which shows that the adhesion force of a finite scale nanofilm, e.g., the gecko's spatula, is strongly reduced at an intermediate roughness. The results obtained here with a finite nanofilm in contact with a rough surface can qualitatively reveal how geckos adapt to various rough surfaces and why geckos overbuild the number of setae [28].

Figure 5 shows the normalized effective interfacial energy as a function of the surface roughness for two kinds of wavelengths; one is less than the length of a nanofilm, and the other is larger than the length of a nanofilm and different thicknesses of nanofilms, respectively. The other parameters are the same as those taken in Fig. 3. One can see that the thickness of a nanofilm shows a significant influence 


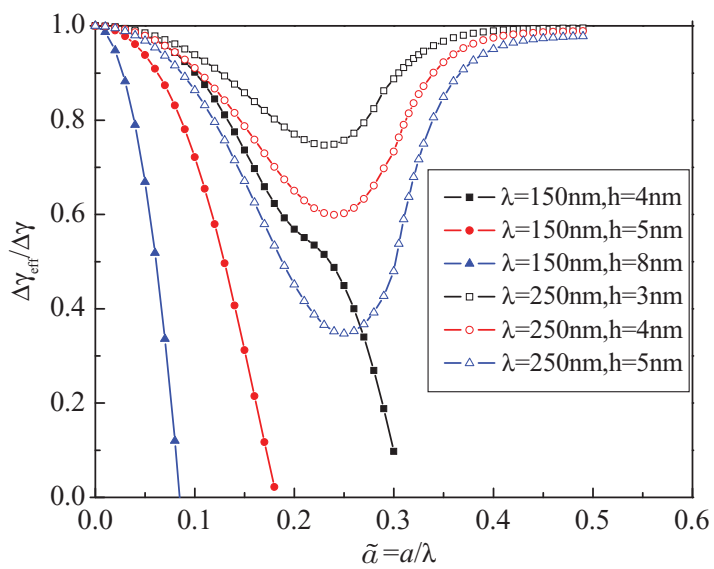

FIG. 5. (Color online) The relation between the normalized effective interfacial energy $\Delta \gamma_{\text {eff }} / \Delta \gamma$ and the surface roughness $a / \lambda$ for cases with different wavelengths $\lambda$ and different film thicknesses $h$.

on the adhesion of a nanofilm in adhesive contact with a rough surface. It is shown that the effective interfacial energy decreases with increasing thickness at a given surface roughness $a / \lambda$ not only in the case with a wavelength less than the length of the nanofilm, but also in the one with a wavelength larger than the length of the nanofilm. If the film is thick enough, the stored bending energy is so large that it can consume the interfacial energy, and as a result, the film will detach from the substrate very easily. When the film is thicker than the critical size, the elastic strain energy is larger than the adhesion energy; the film will detach from the rough substrate spontaneously and, thus, partial contact will occur [16]. With this in mind, we wonder whether an optimal thickness exists for a nanofilm to adapt to both rough and flat surfaces.

Considering the effect of film thickness on adhesion energy, one can see that the adhesion energy is actually a function of the nanofilm's thickness and almost remains constant when the film thickness is larger than $15 \mathrm{~nm}$ as shown in Fig. 6(a), where the attractive energy is denoted as negative. Figure 6(b) shows the normalized effective interfacial energy as a function of surface roughness for two cases, one is with a constant interfacial energy $\Delta \gamma$, the other is with a size-dependent interfacial energy $\Delta \gamma(h)$ From Fig. 6(b), one can see variations of the effective interfacial energy with the surface roughness, which in both cases, have the same trend, and only the effective interfacial energy, considering the size effect, slightly reduces at a given surface roughness, compared to the case with a constant interfacial energy.

According to Eqs. (15) and (17), the nondimensional adhesion energy and adhesion force, as a function of the film thickness with the other parameters similar to a gecko's spatula for a finite-size nanofilm in adhesive contact with a flat surface, are shown in Figs. 7(a) and 7(b). One can see that the adhesion energy, as a function of film thickness as shown in Fig. 7(a), is similar to that derived from thermodynamics [Fig. 6(a)]. Similar to Ref. [20], we take the separating distance $D=0.3 \mathrm{~nm}$, and the corresponding adhesion force approximately saturates when the thickness is about $5 \mathrm{~nm}$ [see the inset in Fig. 7(b)], which is almost identical to the thickness of a real spatula in a geckos' foot. However, it has
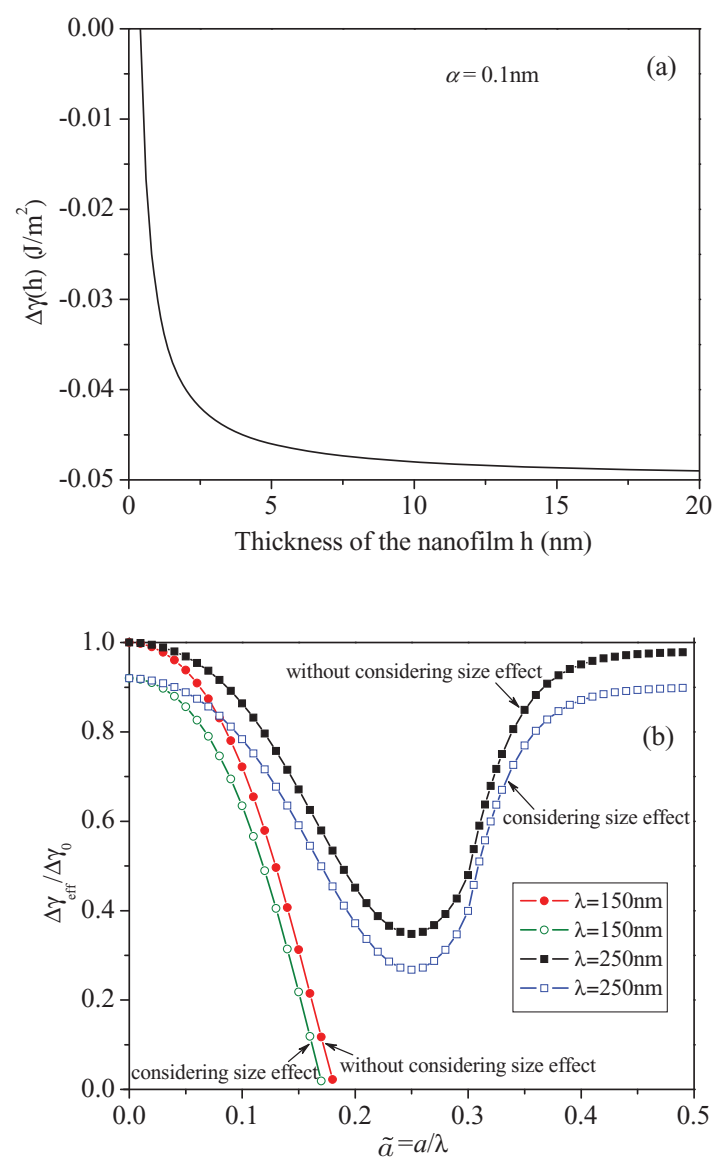

FIG. 6. (Color online) (a) The effect of film thickness on adhesion energy. (b) The relation between the normalized effective interfacial energy $\Delta \gamma_{\text {eff }} / \Delta \gamma_{0}$ and the surface roughness $a / \lambda$ for the case with size-dependent adhesion energy and the one with constant adhesion energy.

been suggested that the distance of $50 \mathrm{~nm}$, before retarded van der Waals forces, needs to be considered [40,41]. Actually, only the normal or nonretarded van der Waals forces (the interaction energy demonstrated by the L-J potential) are considered in the present paper, and according to the previous experimental measurement [40], the range of nonretarded van der Waals forces is smaller than $12 \mathrm{~nm}$. One may wonder whether such a thin spatula with a thickness of about $5 \mathrm{~nm}$ is strong enough to support a gecko's body weight. Experimental observations have shown that the adhesive system of the gecko is a hierarchical structure. One foot of the gecko has five toes, each toe with several rows of sticky lamellae, each lamella with many seta arrays consisting of thousands of setae, which amounts to about 200000 setae per toe, and each seta consisting of 100-1000 spatulas at its end [20,28]. Assuming each seta consists of 100 spatulas and the upper bounded body weight of a gecko is about $100 \mathrm{~g}$, thus, the force of each spatula supported is about $50 \mathrm{nN}$. According to Gao and Chen [42], a typical estimate of the theoretical strength can range between $1 \%$ and $10 \%$ of the Young's modulus. If we take $E=2 \mathrm{GPa}$, the width of a spatula is $200 \mathrm{~nm}$, and $\sigma_{\text {th }}=5 \% E$; the minimal thickness of the gecko spatula pad that can support the body weight is about $2.5 \mathrm{~nm}$, which guarantees the failure occurring at the adhesive interface rather than due to the fracture of 

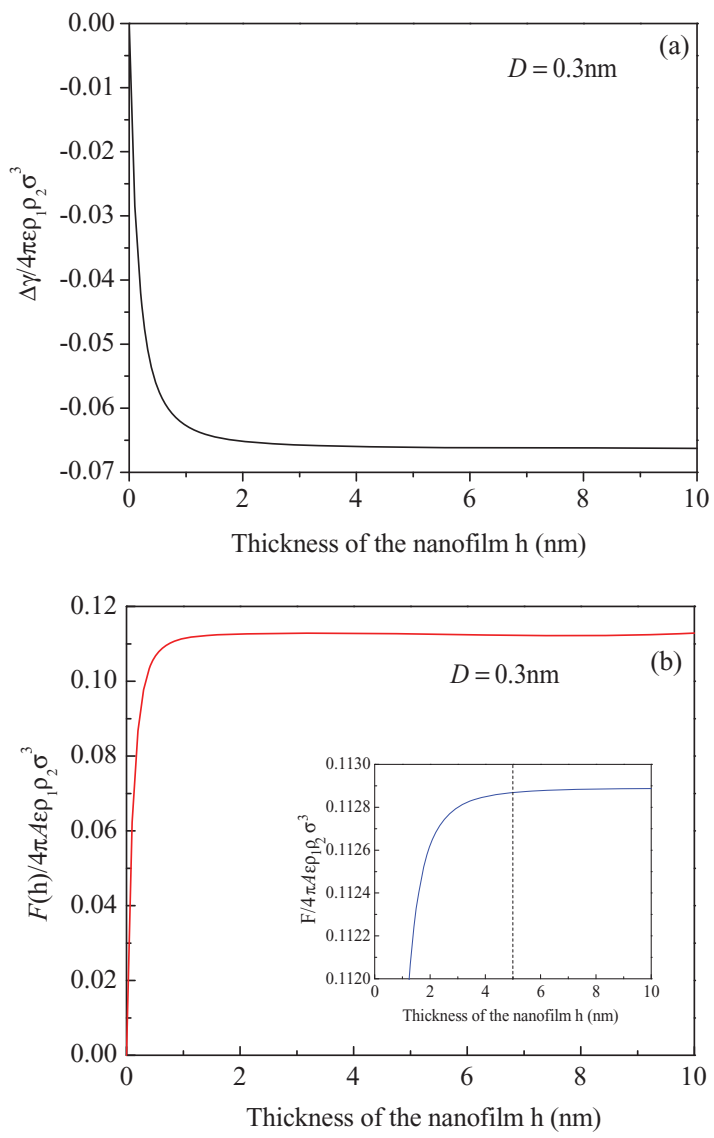

FIG. 7. (Color online) The effect of film thickness on (a) the adhesion energy and (b) the corresponding adhesion force in a model of a finite-size nanofilm in adhesive contact with a flat semi-infinite space. The distance between them is $0.3 \mathrm{~nm}$. The inset is given in order to find the critical thickness after which the adhesion force will saturate.

the spatula pad itself. Therefore, the thickness of the geckos' spatula pad, $5 \mathrm{~nm}$, is a proper thickness not only to adapt to the rough surface and to saturate the adhesion force on a flat surface, but also to resist the fracture of the spatula pad itself.

From the above results obtained in the normal pull-off model, one may wonder if the results are able to apply to the real peeling case. When a spatula detaches from a substrate, a shear force also is applied, thus, the preloading [21] and peeling directions $[22,43]$ may play an important role in the gecko detachment. In fact, when a gecko's spatula adheres to a flat substrate, the above normal pull-off force can be viewed as the available maximum normal adhesion force of a pulled spatula [20]. While in the case of a rough substrate, an effective interfacial energy is introduced, and the peeling force can quickly be obtained by replacing the interfacial energy with the effective interfacial energy in Kendall's peeling model [16] as shown in Eq. (1). Furthermore, only the normal adhesion force is usually obtained in most experimental measurements of the gecko's spatulas adhesion force $[17,30,44]$.

\section{CONCLUSION}

A model of a finite-size bioinspired nanofilm in contact with a sinusoidal rough substrate was presented in this paper. It was found that the influence of surface roughness on adhesion significantly depended on the geometrical parameters of the surface roughness, such as the wavelength and the amplitude of the roughness. When the length of a nanofilm is larger than the wavelength of the surface roughness, the adhesion force will decrease with increasing surface roughness, while the adhesion force decreases initially and then increases when the length of the nanofilm is smaller than the wavelength. The present results agree well with a recent experimental observation on adhesion between a gecko's spatula and a rough surface [17].

For a determined roughness of a surface, the effective interfacial energy and the adhesion force would increase with decreasing thickness of a finite-size nanofilm due to the consumption of film-bending strain energy. However, on a flat surface, the adhesion force increases with an increasing thickness of the nanofilm and saturates when the thickness is about $5 \mathrm{~nm}$. On the other hand, the thickness of a gecko's spatula cannot be too thin to support its body weight, which requires a minimal value about $2.5 \mathrm{~nm}$. In contrast to the real size of a gecko's spatula, one can infer that overbuilding of the number of the geckos' setae may be required to overcome the reduced adhesion due to surface roughness in some cases, and the real thickness, $5 \mathrm{~nm}$, of a gecko's spatula may be an optimal one to compromise all the requirements, such as adhesion on a flat surface, adhesion on an arbitrary rough surface, and supporting its body weight. Furthermore, although the roughness of the substrate in the present paper is assumed to be a sinusoidal function, the model can readily be extended to the case with a stochastically rough surface.

\section{ACKNOWLEDGMENTS}

The work reported here is supported by the NSFC through Grants No. 10972220, No. 10732050, and No. 11021262. In addition, the authors thank the two anonymous reviewers for their helpful and professional suggestions.
[1] K. N. G. Fuller and D. Tabor, Proc. R. Soc. London Ser. A 345, 327 (1975).

[2] K. L. Johnson, K. Kendall, and A. D. Roberts, Proc. R. Soc. London, Ser. A 324, 301 (1971).

[3] G. A. D. Briggs and B. J. Briscoe, J. Phys. D 10, 2453 (1977).

[4] K. N. G. Fuller and A. D. Roberts, J. Phys. D 14, 221 (1981).
[5] G. A. D. Briggs and B. J. Briscoe, Nature (London) 260, 313 (1976).

[6] Y. I. Rabinovich et al., J. Colloid Interface Sci. 232, 10 (2000).

[7] Y. I. Rabinovich et al., J. Colloid Interface Sci. 232, 17 (2000).

[8] B. N. J. Persson and E. Tosatti, J. Chem. Phys. 115, 5597 (2001).

[9] B. N. J. Persson, Phys. Rev. Lett. 89, 245502 (2002). 
[10] G. Palasantzas and J. T. M. De Hosson, Phys. Rev. E 67, 021604 (2003).

[11] G. Palasantzas and J. T. M. De Hosson, J. Appl. Phys. 94, 3041 (2003).

[12] Z. L. Peng, S. H. Chen, and A. K. Soh, Int. J. Solids Struct. 47, 1952 (2010).

[13] J. A. Greenwood and J. B. Williams, Proc. R. Soc. London, Ser. A 295, 300 (1966).

[14] B. N. J. Persson and S. Gorb, J. Chem. Phys. 119, 11437 (2003).

[15] B. N. J. Persson et al., J. Phys.: Condens. Matter 17, R1 (2005).

[16] C. Majidi and R. S. Fearing, Proc. R. Soc. London Ser. A 464, 1309 (2008).

[17] G. Huber et al., Acta Biomater. 3, 607 (2007).

[18] S. Gorb, Attachment Devices of Insect Cuticle (Kluwer Academic, Dordrecht, 2001).

[19] A. Peressadko and S. Gorb, in First International Industrial Conference Bionik, edited by I. Boblan and R. Bannasch (German Federal Ministry of Education, Hannover, Germam, 2004), p. 257.

[20] Y. Tian et al., Proc. Natl. Acad. Sci. USA 103, 19320 (2006).

[21] B. Chen, P. D. Wu, and H. J. Gao, J. R. Soc. Interface 6, 529 (2009).

[22] N. S. Pesika et al., J. Adhes. 83, 383 (2007).

[23] K. Kendall, J. Phys. D 8, 1449 (1975).

[24] S. Zilberman and B. N. J. Persson, Solid State Commun. 123, 173 (2002).

[25] J. L. Liu et al., J. Phys.-Condes. Matter 19, 356002 (2007).
[26] R. C. Tolman, J. Chem. Phys. 17, 333 (1949).

[27] C. H. Chiu and H. J. Gao, Thin Films 356, 33 (1995).

[28] K. Autumn et al., Nature (London) 405, 681 (2000).

[29] K. Autumn et al., Proc. Natl. Acad. Sci. USA 99, 12252 (2002).

[30] G. Huber et al., Biol. Lett. 1, 2 (2005).

[31] J. Israelachvili, Intermolecular and Surface Forces (Academic, London, 1991).

[32] N. Yu and A. A. Polycarpou, J. Colloid Interface Sci. 278, 428 (2004).

[33] T. W. Kim and B. Bhushan, J. R. Soc. Interface 5, 319 (2008).

[34] C. Argento, A. Jagota, and W. C. Carter, J. Mech. Phys. Solids 45, 1161 (1997).

[35] G. J. Ackland and V. Vitek, Phys. Rev. B 41, 10324 (1990).

[36] M. I. Baskes, Phys. Rev. B 46, 2727 (1992).

[37] M. I. Baskes and R. A. Johnson, Model. Simul. Mater. Sci. Eng. 2, 147 (1994).

[38] K. W. Jacobsen, J. K. Norskov, and M. J. Puska, Phys. Rev. B 35, 7423 (1987).

[39] J. Israelachvili and D. Tabor, Proc. R. Soc. London, Ser. A 331, 19 (1972).

[40] J. Israelachvili and D. Tabor, Nature (London) Phys. Sci. 236, 106 (1972).

[41] F. W. Delrio et al., Nature Mater. 4, 629 (2005).

[42] H. J. Gao and S. H. Chen, J. Appl. Mech. 72, 732 (2005).

[43] B. X. Zhao et al., Langmuir 24, 1517 (2008).

[44] W. X. Sun et al., Biophys. J. 89, L14 (2005). 